\title{
ESTADO NUTRICIONAL DE PACIENTES \\ HOSPITALIZADOS EN MEDICINA INTERNA EN UN HOSPITAL DE REFERENCIA
}

Indhira Patricia Arana Montoya MD*, William Rojas García MD**

\section{Resumen}

Introducción: la malnutrición es frecuente en pacientes hospitalizados, asociándose con malos resultados clínicos, complicaciones y estancias prolongadas. Objetivo: determinar la prevalencia de la malnutrición en el paciente hospitalizado en el servicio de medicina interna. Métodos: estudio descriptivo que incluyó pacientes mayores de 18 años durante las primeras 72 horas de hospitalización; se excluyeron enfermos críticos. El estado nutricional hospitalario se evaluó con la escala de valoración global subjetiva, previa firma de consentimiento informado por el paciente o su familiar. Se recolectó información acerca de estrato social, estancia hospitalaria, edad, sexo y comorbilidades. Resultados: se evaluaron 258 pacientes hospitalizados. La prevalencia de malnutrición fue 47,7\%, con alta frecuencia en mayores de 68 años. EI 41,4\% de los malnutridos ingresaron al hospital por infecciones. El promedio de estancia fue siete días en los pacientes con y sin desnutrición, pero la estancia mayor de 25 días sólo se observó en los primeros. No hubo diferencia en el estado nutricional en pacientes con comorbilidades metabólicas y pulmonares. Conclusión: la prevalencia de malnutrición en los pacientes del servicio de medicina interna del Hospital de San José es cercana a la encontrada en el estudio latinoamericano (ELAN, 50,2\%). La estancia hospitalaria mayor a 25 días se observó sólo en el grupo de pacientes malnutridos, como se informa en otros estudios.

Palcbras clave: malnutrición, hospitalizados, medicina interna, desnutrición hospitalaria.

Abreviaturas: MN, malnutrición: VGS, valoración global subjetiva.

\section{NUTRITIONAL STATUS OF PATIENTS ADMITTED TO A REFERRAL INTERNAL MEDICINE SERVICE}

\section{Abstract}

Introduction: malnutrition is highly prevalent among hospitalized patients and is associated with poor clinical outcomes, complications and a longer hospital stay. Objective: to determine the prevalence of malnutrition on patients admitted to the internal medicine service. Methods: this is a descriptive study including all patients 18 years-of-age or older admitted to the internal medicine service and screened within 72 hours of admission; critical patients were excluded. Nutritional status was determined using the Subjective Global Assessment (SGA). An informed consent was previously

Fecha recibido: marzo 8 de 2010 Fecha aceptado: agosto 27 de 2010

* Residente II de Endocrinología, Fundación Universitaria de Ciencias de la Salud, Hospital de San José, Bogotá DC. Colombia.
** Jefe del Servicio de Endocrinología, Hospital de San José. Profesor Asociado Fundación Universitaria de Ciencias de la Salud. Bogotá DC. Colombia. 


\begin{abstract}
signed by the patient or one of his relatives. Data regarding social status, hospital stay, age, sex and comorbidities were obtained. Results: 258 hospitalized patients were assessed. The prevalence of malnutrition was $47.7 \%$, with a high frequency in patients older than 68 years. Infection was the reason for admittance in $41.4 \%$ of malnourished patients. The mean hospital stay was 7 days in malnourished and non malnourished patients, but the longest, a 25-day stay, was observed in those with malnutrition. No difference in nutritional status was observed in patients with metabolic or pulmonary comorbidities. Conclusion: the prevalence of malnutrition in patients admitted to the Internal Medicine Service at Hospital de San José is close to that reported in the Latin American Nutrition Study (ELAN results, 50.2\%). A hospital stay greater than $\mathbf{2 5}$ days was observed only in the malnourished patient group and is similar to that reported in other trials.
\end{abstract}

Key: Words: malnutrition, hospitalized patients, internal medicine, hospital malnutrition

\section{Introducción}

La MN es un trastorno metabólico causado por el desequilibrio entre la ingesta de nutrientes y las necesidades corporales. Es prevalente en países como el nuestro en vía de desarrollo, causada por la carencia de alimentos en cantidad y calidad suficiente (MN primaria). ${ }^{\prime}$ En las sociedades industrializadas la $\mathrm{MN}$ suele estar relacionada con alguna enfermedad que altera la ingesta, el metabolismo o la absorción de los nutrientes (MN secundaria) y se encuentra con frecuencia en pacientes hospitalizados o institucionalizados. ' La MN hospitalaria como causa de inadecuada respuesta al tratamiento fue descrita hace más de 30 años por Butterworth ${ }^{2}$ en su artículo El esqueleto en el closet, cuando llamó la atención de la comunidad médica sobre la malnutrición intrahospitalaria. Vinieron entonces grupos interesados en lograr evaluar el estado nutricional del paciente hospitalizado en forma práctica y confiable.

Baker en 1982 introdujo la escala de VGS con el fin de evaluar el estado nutricional en la cama del paciente sin necesidad de precisar el análisis de composición corporal. Esta escala fue modificada y validada por Detsky y colaboradores. ${ }^{3}$ El instrumento se ha aplicado en estudios como el ELAN ${ }^{4}$ en el cual se incluyeron trece países de Latinoamérica (Argentina, Brasil, CostaRica, Cuba, Chile, México, Panamá, Paraguay, Perú, Puerto Rico, República Dominicana, Uruguay y Venezuela) que incluyeron 9.348 pacientes hospitalizados y reportaron una prevalencia de MN hospitalaria de 50,2\%. Previo a éste se realizó el IBANUTRI $^{5}$ estudio brasilero de nutrición hospitalaria que incluyó 4.000 pacientes de 25 hospitales en doce estados, encontrandouna prevalencia de MNhospitalaria de $48,1 \%$, con una mediana de hospitalización de nueve días en pacientes malnutridos, mayor que en los bien nutridos. Éstos, junto con el estudio argentino publicado por Baccaro y Correia son los más importantes realizados en Latinoamérica que han utilizado la VSG. ${ }^{6,7}$

La MN en el paciente hospitalizado se ha asociado con estancias prolongadas, aumento de mortalidad, morbilidad y de los costos. ${ }^{7,8,9}$ Hay múltiples factores que podrían afectar el estado nutricional en donde la enfermedad actual ocupa el primer lugar de riesgo. Otros elementos que suelenasociarse son el nivel socioeconómico, la edad y la patología asociada, según lo reportado por ELAN. ${ }^{4}$ El estudio realizado en 1987 por Detsky y col. en pacientes posquirúrgicos, reveló un incremento de $10 \%$ en las complicaciones, en especial procesos infecciosos. ${ }^{10} \mathrm{En}$ nuestro estudio se buscó describir la prevalencia de $\mathrm{MN}$ hospitalaria en el paciente médico hospitalizado y observar si otras condiciones como el estrato social, el diagnóstico de ingreso y las enfermedades asociadas afectan la frecuencia de presentación de desnutrición.

\section{Métodos}

Se realizó un estudio descriptivo entre los meses de marzo a julio de 2009. Se evaluaron pacientes del servicio de medicina interna dentro de las primeras 72 horas de hospitalización. Se incluyeron los mayores de 18 años y se excluyeron aquellos con trastornos psiquiátricos (psicosis, anorexia nerviosa, bulimia o depresión registrados en la historia clínica), los críticos (procedentes de la UCI o que estaban por ingresar a ella), o con estadios avanzados de enfermedad terminal definida 
(cáncer, VIH, neurológicas) y mujeres gestantes (referido por la paciente o por la historia).

El estado nutricional fue evaluado mediante la escala de VGS que incluye cambios en el peso, la ingesta diaria, síntomas gastrointestinales, capacidad funcional, diagnóstico clínico y tres parámetros subjetivos del examen físico que valoran el estado nutricional como (A) bien nutrido, (B) moderadamente malnutrido y (C) MN severa. ${ }^{3}$

La evaluación fue realizada por residentes de endocrinología y medicina interna, y dos internos del servicio de endocrinología. Los evaluadores no realizaron seguimiento de la hospitalización. La estancia se tomó del registro de altas del hospital y se presenta en días. Además se recolectó información acerca de la edad, sexo, estrato socioeconómico y enfermedades asociadas. No se valoró la mortalidad en este estudio. Los diagnósticos de ingreso se agruparon de acuerdo con el sistema comprometido o la condición clínica prevalente (infección o cáncer), así como las comorbilidades. Los datos se analizaron en STATA 10 y se calcularon medidas de tendencia central, de dispersión y frecuencias.

\section{Resultados}

Se analizaron todos los pacientes incluidos en el estudio (258 hospitalizados en el servicio de medicina interna), 109 $(42,2 \%)$ hombres y $149(57,8 \%)$ mujeres, con un prome- dio de edad de 60 años y desviación estándar de 19.7 años. La prevalencia de $\mathrm{MN}$ encontrada en esta población fue 47,7\%; severa en 8,1\% (21) y moderada en 39,5\% (102). En la Tabla 1 se describen los datos demográficos, en los cuales el $90 \%$ de los pacientes están en estrato socioeconómico medio bajo y bajo (de uno a tres).

La mayoría de los pacientes con diagnóstico de malignidad al ingreso se encontraron mal nutridos, contrario a lo que ocurre con aquellos que la presentaron como comorbilidad. Los malnutridos sin comorbilidades fueron $16 \%$ y por lo regular cursaban con procesos infecciosos. La enfermedad cardiovascular fue la comorbilidad más frecuente pero no se encontró diferencia en el estado nutricional de estos pacientes (Tabla 2). Los malnutridos hospitalizados por procesos infecciosos fueron $20 \%$ del total de la población.

En el análisis se observó mayor frecuencia de malnutrición en mayores de 68 años (Tabla 3). La estancia hospitalaria más prolongada (mayor de 25 días) se presentó sólo en pacientes malnutridos, aunque el promedio de días de hospitalización fue cinco para desnutridos y bien nutridos (Tabla 3).

\section{Discusión}

La literatura mundial resalta la importancia de la nutrición en la recuperación y calidad de vida de los pacientes hospitalizados utilizando la VGS ${ }^{11}$, en especial en

\begin{tabular}{|l|c|c|c|}
\hline \multicolumn{3}{|c|}{ Tabla I. Estado nutricional según la escala de VGS. } \\
\hline Características & $\begin{array}{c}\text { Bien nutrido } \\
n=135\end{array}$ & $\begin{array}{c}\text { Malnutrición moderada } \\
n=102\end{array}$ & Malnutrición severa \\
& $n=21$
\end{tabular}

*Años, $\ddagger$ días, RIQ † rango intercuartílico. 
Tabla 2. Estado nutricional relacionado con comorbilidades y diagnóstico de ingreso

\begin{tabular}{|l|c|c|}
\hline Caracteristicas n (\%) & Bien nutridos (135) & Malnutrición (I 23) \\
\hline Comorbilidades (SI) & & $60(48,8)$ \\
Cardiovasculares & $59(43,7)$ & $37(30)$ \\
Pulmonares & $27(20)$ & $17(13.8)$ \\
Renales & $8(5.9)$ & $22(17.9)$ \\
Metabólicas & $24(17.7)$ & $8(6,5)$ \\
Reumatológicas & $14(10.3)$ & $8(6,5)$ \\
Malignas & $8(5.9)$ & $16(13)$ \\
Ninguna & $32(23.7)$ & $4(3.2)$ \\
Otras* & $3(2.2)$ & $51(41.4)$ \\
Diagnóstico de ingreso & & $17(13.8)$ \\
Infecciosas & $54(40)$ & $11(8.9)$ \\
Cardíacas & $23(17)$ & $17(13.8)$ \\
Cáncer & $3(2.2)$ & $2(1.6)$ \\
Pulmonar crónica & $17(12.6)$ & $5(4)$ \\
Diabetes & $6(4.4)$ & $8(6.5)$ \\
Renal & $1(0.7)$ & $12(9.7)$ \\
Digestiva & $11(0.7)$ & $20(1.49)$ \\
Otrost & & \\
\hline
\end{tabular}

* Neurológicas no vasculares, hematológicas benignas; † neurológico, intoxicación medicamentosa, hematológicas benignas.

\begin{tabular}{|l|c|c|}
\hline \multicolumn{3}{|c|}{ Tabla 3. Estado nutricional según edad y } \\
estancia hospitalaria (días) \\
\hline Características n (\%) & Malnutrición \\
\hline Grupos edad (años) & NO (135) & SI (123) \\
18-47 & $46(34.2)$ & $26(21.2)$ \\
$48-67$ & $45(33.3)$ & $30(24.4)$ \\
Mayores de 68 & $44(32.5)$ & $57(46.4)$ \\
Estancia (días ) & & \\
I-8 & $114(84.4)$ & $88(71.5)$ \\
$9-16$ & $15(11.1)$ & $25(20.4)$ \\
17-24 & $6(4.5)$ & $4(3.2)$ \\
Mayores de 25 & 0 & $6(4.9)$ \\
\hline
\end{tabular}

poblaciones específicas como los adultos mayores, niños, enfermos con cáncer, afección crítica, lesión renal crónica y trastornos gastrointestinales. ${ }^{7,8,12-16}$ Sin embargo, a la fecha no se conocen estudios publicados en nuestro país que analicen esta problemática. Con esta investigación se pretendió describir las características de los pacientes hospitalizados en medicina interna y la prevalencia de MN por medio de VSG.

El hospital donde se realizó el estudio es un centro de referencia, nivel III de atención, donde se reciben pacientes con patología de alta complejidad, lo que indica enfermedades graves que con frecuencia requieren terapias e intervenciones agresivas. Estos factores predisponen al desarrollo de malnutrición y pueden explicar que en este tipo de centros su prevalencia sea alta. ${ }^{17}$

La mayor parte de la población que acudió al hospital fue de estrato socioeconómico medio o bajo y ninguno del alto. Aunque esto refleja que la mayoría de los pacientes con $\mathrm{MN}$ severa pertenezcan a estos niveles socioeconómicos, no se puede afirmar que el estrato afecte el estado nutricional del paciente hospitalizado, a diferencia de lo aseverado en los estudios IBANUTRI ${ }^{6}$ y ELAN $^{5}$ donde relacionan la situación socioeconómica con el estado nutricional considerándolo como factor de riesgo en MN hospitalaria. Esto indica la importancia de profundizar en la influencia del nivel socioeconómico y la MN hospitalaria aún en estratos altos.

La frecuencia de desnutrición hospitalaria encontrada en este estudio fue de $47,7 \%$, similar a las investigaciones realizadas en Latinoamérica como IBANUTRI que reporta una prevalencia de $48,1 \%{ }^{6}$ y $E_{L A N}{ }^{5}$ con $50,2 \%$, aunque no incluyó a Colombia. Sin embargo, estas cifras son altas comparadas con los reportes de $\mathrm{MN}$ en publicaciones europeas, por ejemploEspaña ${ }^{17}$ informa $40,2 \%$, Alemania $32 \%{ }^{18}$ y el Reino Unido de $44 \%$. ${ }^{19}$ Conocien- 
do la alta prevalencia de $\mathrm{MN}$ y sus efectos sobre la evolución de los pacientes, el personal asistencial y administrativo en los hospitales debe hacerse partícipe de intervenir en la malnutrición hospitalaria como un gran apoyo en la recuperación de sus pacientes.

La comorbilidad más frecuente fue cardiovascular (incluye patologías cerebrovasculares), sin distribución diferente entre los nutridos y malnutridos, pero la prevalencia de $\mathrm{MN}$ en este grupo fue mayor $(48,8 \%)$ que lo reportado por IBANUTRI $(36 \%) .{ }^{6}$ La literatura médica menciona que las enfermedades cardiovasculares son la segunda complicación más frecuente después de las infecciosas. ${ }^{7}$ La mayoría de pacientes con enfermedad pulmonar crónica presentó malnutrición, dado posiblemente por su condición que dificulta la ingesta de alimentos y disminuye el apetito. La frecuencia está por debajo de lo observado en IBANUTRI con $67,4 \%,{ }^{6}$ que reporta mayor $\mathrm{MN}$ en pacientes con patologías hematológicas y autoinmunes; en contraste en nuestro estudio estas comorbilidades fueron las menos frecuentes, similar a lo publicado por Vidal ${ }^{17}$ que no observó diferencia en malnutrición en las enfermedades crónicas.

La estancia hospitalaria fue similar en los pacientes mal nutridos y bien nutridos, pero las estancias prolongadas (más de 25 días) sólo se observaron en el grupo de $\mathrm{MN}$ como se ha descrito en las publicaciones de Vidal y Baccaro, aunque ellos mencionan que esta estancia se correlaciona con $\mathrm{MN} .^{12,13,17}$ Por otra parte, la estancia prolongada se ha considerado factor de riesgo en $\mathrm{MN}$ hospitalaria ${ }^{4,5,14,20}$, lo cual no fue evaluado en el presente estudio. El 46\% de los pacientes malnutridos son mayores de 68 años, lo cual puede estar relacionado como lo describen IBANUTRI, ELAN y Correia que reportan que los pacientes mayores de 60 años presentan mayor riesgo de desarrollar MN hospitalaria. ${ }^{4,5,7}$ Es importante resaltar que el $16 \%$ sin comorbilidades y malnutridos, cursaban por lo regular con procesos infecciosos como diagnóstico de ingreso. La infección es la causa de hospitalización más frecuente, pero la distribución entre bien nutridos y mal nutridos fue similar. Baccaro y colaboradores reportan que el $38,6 \%$ de los pacientes ingresaron por este diagnóstico, y tienen OR de 2.31 para malnutrición. ${ }^{6}$

La VGS es un instrumento sencillo y ágil comparado con otros métodos pues facilita la evaluación del estado nutricional en forma confiable sin aplicar medidas invasivas y además es de bajo costo. Este último aspecto puede ser relevante para que instituciones como la nuestra consideren implementar el uso de esta herramienta y así generar cultura hospitalaria frente a la importancia del estado nutricional de los pacientes, que al impactar en los tratamientos y la estancia hospitalaria, eleva los costos.

En esta investigación faltó evaluar la mortalidad porque no se realizó seguimiento directo al paciente. No obstante, es una variable que está relacionada con el impacto de la MN hospitalaria mencionada en estudios como el argentino y el español, que refieren mayor frecuencia de MN en los fallecidos. ${ }^{8,11,20}$

\section{Referencias}

1. Bhasin S. Malnutrition and assessment. En Harrisons principles of internal medicine. 17 ed. United states of America: Mc graw- Hill; 2008.

2. Butterworth CE, Jr. The skeleton in the hospital closet. Nutrition. 1994; $10(5): 442$.

3. Detsky AS, McLaughlin JR. et al. What is subjective global assessment of nutritional status?. JPEN J Parenter Enteral Nutr. 1987; 11(1): 8-13.

4. Correia MI; Campos AC. Prevalence of hospital malnutrition in Latin America: the multicenter ELAN study. Nutrition. 2003; 19(10): 823-5.

5. Waitzberg DL, CaiaffaWT, et al. Hospital malnutrition: the Brazilian national survey (IBRANUTRI): a study of 4000 patients. Nutrition. 2001; 7(7-8): 573-80.

6. Baccaro F, Moreno JB, et al. Subjective global assessment in the clinical setting. JPEN J Parenter Enteral Nutr. 2007; 31(5):406-9.

7. Correia MI, Waitzberg DL. The impact of malnutrition on morbidity, mortality, length of hospital stay and costs evaluated through a multivariate model analysis. Clin Nutr. 2003; 22(3):235-9.

8. Covinsky KE, Martin GE, etal. The relationship between clinical assessments of nutritional status and adverse outcomes in older hospitalized medical patients. J Am Geriatr Soc. 1999; 47(5): 532-8.

9. Valentini L, Schaper L, et al. Malnutrition and impaired muscle strength in patients with Crohn's disease and ulcerative colitis in remission. Nutrition. 2008; 24(7-8): 694-702.

10. Detsky AS, Baker JP, et al. Predicting nutrition-associated complications for patients undergoing gastrointestinal surgery. JPEN J Parenter Enteral Nutr. 1987; $11(5)$ : 440-6.

11. Norman $\mathrm{K}$, Kirchner $\mathrm{H}$, et al. Malnutrition affects quality of life in gastroenterology patients. World J Gastroenterol. 2006; 12(21): 3380-5.

12. Atalay BG, Yagmur C, et at. Use of subjective global assessment and clinical outcomes in critically ill geriatric patients receiving nutrition support. JPEN J Parenter Enteral Nutr. 2008 Jul; 32(4): 454-9. 
13. Barbosa-Silva MC; Barros AJ. Indications and limitations of the use of subjective global assessment in clinical practice: an update. Curr Opin Clin Nutr Metab Care. 2006; 9(3):2 63-9.

14. Keith JN. Bedsidenutritionassessment past, present, and fiture: a review of the Subjective Global Assessment. Nutr Clin Pract. 2008; 23(4): 410-6.

15. Secker DJ, Jeejeebhoy KN. Subjective Global NutritionalAssessment for children. Am J Clin Nutr. 2007; 85(4): 1083-9.

16. Kubrak C, Jensen L. Critical evaluation of nutrition screening tools recommended for oncology patients. Cancer Nurs. 2007; 30(5): E1-E6.
17. Vidal A; Iglesias MJ, et al. Prevalence of malnutrition in medical and surgical wards of a university hospital. Nutr Hosp. 2008; 23(3): 263-7.

18. Weinsier RL, Hunker EM, et al. Hospital malnutrition. A prospective evaluation of general medical patients during the course of hospitalization. Am J Clin Nutr. 1979; 32(2): 418-26.

19. Lamb CA, Parr J., et al. Adult malnutrition screening, prevalence and management in a United Kingdom hospital: cross-sectional study. $\mathrm{Br} \mathrm{J}$ Nutr. 2009; 102(4): 571-5.

20. Waitzberg DL, Correia MI. Nutritional assessment in the hospitalized patient. Curr Opin Clin Nutr Metab Care. 2003; 6(5): 531-8

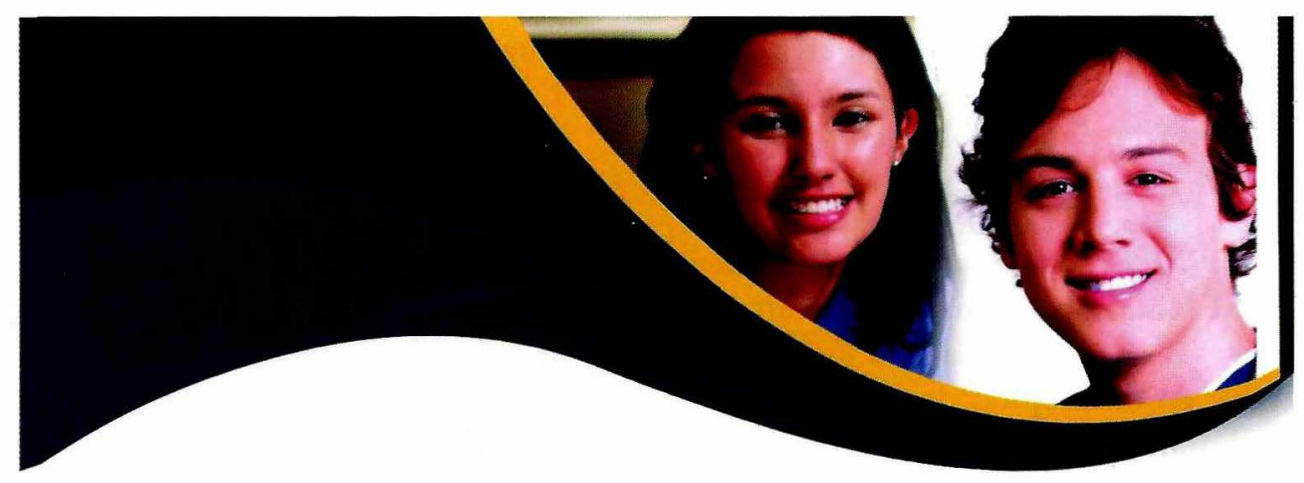

\section{Fundación Universitaria de Ciencias de la Salud - FUCS}

Contamos con dos hospitales propios para la formación de nuestros estudiantes: Hospital de San José y Hospital Infantil Universitario de San José

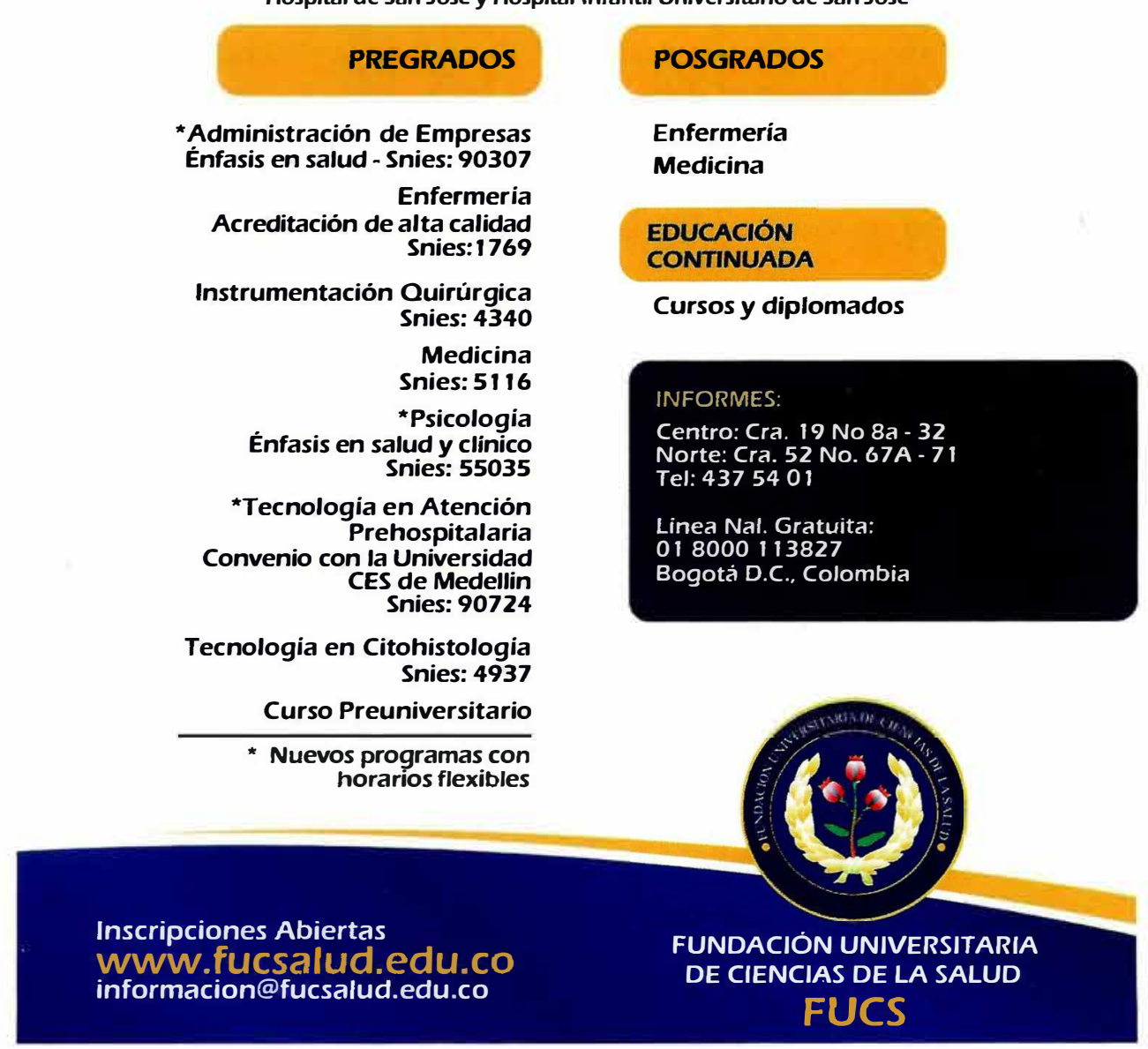

